\title{
Incommensurate phases in ferromagnetic spin-chains with weak antiferromagnetic interchain interaction Chain
}

\author{
C. Pich \\ Department of Physics, University of California, Santa Cruz, CA 95064 \\ F. Schwabl \\ Technische Universiät München, James-Franck-Str., 85747 München, Germany
}

(November 15, 2018)

\begin{abstract}
We study planar ferromagnetic spin-chain systems with weak antiferromagnetic inter-chain interaction and dipole-dipole interaction. The ground state depends sensitively on the relative strengths of antiferromagnetic exchange and dipole energies $\kappa=J^{\prime} a^{2} c /\left(g_{L} \mu_{B}\right)^{2}$. For increasing values of $\kappa$, the ground state changes from a ferromagnetic via a collinear antiferromagnetic and an incommensurate phase to a $120^{\circ}$ structure for very large antiferromagnetic energy. Investigation of the magnetic phase diagram of the collinear phase, as realized in $\mathrm{CsNiF}_{3}$, shows that the structure of the spin order depends sensitivly on the direction of the magnetic field in the hexagonal plane. For certain angular domains of the field incommensurate phases appear which are separated by commensurate phases. When rotating the field, the wave vector characterizing the structure changes continuously in the incommensurate phase, whereas in the commensurate phase the wave vector is locked to a fixed value describing a two-sublattice structure. This is a result of the competition between the exchange and the dipole-dipole interaction.
\end{abstract}

\section{INTRODUCTION}

Ferromagnetic spin chains have been studied extensively, experimentally as well as theoretically 8 . Typical systems are $\mathrm{CsNiF}_{3}$ and $\mathrm{RbFeCl}_{3}$ which show quasi onedimensional behavior due to the small lattice constant along the $c$-axis. Nearest neighbor spins couple with a ferromagnetic exchange interaction along the spin chain. A planar anisotropy of the same order of magnitude is found because of the non-vanishing orbital angular momentum of the magnetic ions. Perpendicular to the chain axis the magnetic ions are located on a triangular (hexagonal) lattice with a much larger lattice constant $a$, i.e. the magnetic ions form a simple hexagonal lattice. Thus, at high temperatures a pronounced one-dimensional behavior can be found experimentally. Planar ferromagnets still have rotational symmetry, so that no long range order exists. Nevertheless, spin waves were measured for $\mathbf{q} \neq 0$. At low temperatures the systems undergo a phase transition to a three-dimensional ordered phase for which the antiferromagnetic exchange interaction and the dipole-dipole interaction are responsible. The precise structure of the order in the hexagonal plane depends sensitively on the relative strengths of these two competing interactions. $\mathrm{RbFeCl}_{3}$ has a nearly $120^{\circ}$ structure because the exchange energy is large. In contrast, $\mathrm{CsNiF}_{3}$ has a collinear orientation of the spins due to the large dipole energy. Up to now the three-dimensional ordering has not attracted much interest, but interesting behavior is expected due to the competing interactions which can lead to frustration.

Recently Baehr et al 12 have measured the magnetic excitations in the three-dimensional ordered $\mathrm{CsNiF}_{3}$ $\left(T<T_{N}=2.7 \mathrm{~K}\right)$ by inelastic neutron scattering. For the first time the magnitude of the antiferromagnetic exchange energy in the plane has been determined by measuring the dispersion relation. The important role of the dipole-dipole interaction is manifested by the discontinuity in the magnon energy at the zone center $(\mathbf{q}=0)$. This is an effect of the long range interaction. In this paper we want to study the whole range from vanishing exchange energy up to the region where the dipole energy is small. It will be shown that instead of the frustrated $120^{\circ}$ structure an incommensurate phase will be established. The dispersion relations are evaluated for the commensurate phases. In a second step we investigate the magnetic phase diagram for the collinear phase, especially for $\mathrm{CsNiF}_{3}$, and calculate the critical fields. The $\mathrm{Ni}$ atoms form a simple hexagonal lattice with lattice constants $a \overline{\bar{⿴}} b=6.21 \AA$ and $c=5.2 \AA$ the Landé factor $g_{L}=2.259$ and the spin $S=1$. The outline of the paper is as follows. In chapter II we introduce the model, in chapter III the ground state for vanishing field strength is determined and in chapter IV we study the excitations of the ferromagnetic and the collinear phase. In chapter $\mathrm{V}$ the collinear antiferromagnetic phase with finite fields in the hexagonal plane is investigated. In the appendix we summarize the main relations for the dipole energy.

\section{MODEL}

The starting point of our investigation is the Heisenberg Hamiltonian

$H=-2 J \sum_{i} \mathbf{S}_{i} \mathbf{S}_{i+1}+A \sum_{l}\left(S_{l}^{z}\right)^{2}-$ 


$$
\sum_{\alpha, \beta} \sum_{l, l^{\prime}}\left(J_{l l^{\prime}}^{\prime} \delta^{\alpha \beta}+A_{l l^{\prime}}^{\alpha \beta}\right) S_{l}^{\alpha} S_{l^{\prime}}^{\beta}-g_{L} \mu_{B} \mathbf{H}_{0} \sum_{l} \mathbf{S}_{l} .
$$

Here $J$ denotes the ferromagnetic nearest-neighbor intrachain interaction, $A$ the single-ion anisotropy, $J_{l l^{\prime}}^{\prime}$ the interchain and $A_{l l^{\prime}}^{\alpha \beta}$ the dipole-dipole interaction

$A_{l, l^{\prime}}^{\alpha \beta}=-\frac{\left(g_{L} \mu_{B}\right)^{2}}{2}\left\{\frac{\delta^{\alpha \beta}}{\left|\mathbf{x}_{l}-\mathbf{x}_{l^{\prime}}\right|^{3}}+\frac{3\left(\mathbf{x}_{l}-\mathbf{x}_{l^{\prime}}\right)^{\alpha}\left(\mathbf{x}_{l}-\mathbf{x}_{l^{\prime}}\right)^{\beta}}{\left|\mathbf{x}_{l}-\mathbf{x}_{l^{\prime}}\right|^{5}}\right\}$.

$i$ indicates positions on one and the same spin chain, whereas $l$ indicates all spin positions. The notation for $x_{l}$, used here, is given in the appendix. $\mathbf{H}_{0}$ is an external field perpendicular to the chain axis. We are interested in the case of a planar ferromagnetic chain, i.e. $J, A>0$, which means that the spins are forced to lie in the hexagonal plane perpendicular to the chain. The following calculations are valid only for weak inter-chain interactions, i.e. $J, A \gg J^{\prime},\left(g_{L} \mu_{B}\right)^{2} / a^{2} c$, where $a$ indicates the lattice constant of the triangular lattice in the plane and $c$ the lattice constant of the chain. Because of the long-range of the dipolar interaction, summations over the whole system are performed best by means of the Ewald summation technique 13 (s. appendix). In general, we can differentiate between one-dimensional behaviour at high temperatures due to the large intra-chain energy and the three-dimensional properties at low temperatures.

\section{GROUND STATES FOR $\mathbf{H}_{0}=0$}

First we study the ground state of the threedimensional system without an external field. Due to the pronounced one-dimensional behaviour the classical ground state consists of ferromagnetic spin-chains with the spins ordered in the plane perpendicular to the chains. The spin structure within the hexagonal plane then depends only on the antiferromagnetic exchange and the dipolar interaction. For the following investigation we introduce a dimensionless parameter $\kappa$, which is the ratio of the antiferromagnetic exchange to the dipolar interaction $\left(\kappa_{\mathrm{CsNiF}_{3}}=0.79\right)$ :

$$
\kappa=\frac{J^{\prime} a^{2} c}{\left(g_{L} \mu_{B}\right)^{2}} .
$$

Fourier transformation of the Hamiltonian (Eq. (1)) yields

$$
H=-\sum_{\alpha, \beta} \sum_{\mathbf{q}}\left(J_{\mathbf{q}} \delta^{\alpha \beta}-A \delta^{\alpha z} \delta^{\beta z}+J_{\mathbf{q}}^{\prime} \delta^{\alpha \beta}+A_{\mathbf{q}}^{\alpha \beta}\right) S_{\mathbf{q}}^{\alpha} S_{-\mathbf{q}}^{\beta},
$$

with the nearest-neighbor exchange energies (intrachain and interchain, $J, J^{\prime}>0$ )

$$
\begin{aligned}
& J_{\mathbf{q}}=2 J \cos q_{z} \\
& J_{\mathbf{q}}^{\prime}=-2 J^{\prime}\left(\cos q_{x}+2 \cos \frac{q_{x}}{2} \cos \frac{\sqrt{3} q_{y}}{2}\right) .
\end{aligned}
$$

The classical ground state is determined by the maximal eigenvalue of

$$
V_{\mathbf{q}}^{\alpha \beta}=\left(J_{\mathbf{q}}^{\prime} \delta^{\alpha \beta}+A_{\mathbf{q}}^{\alpha \beta}\right),
$$

with the wave-vector $\mathbf{q}$ constrained to the plane $\left(q_{z}=0\right)$. The eigenvalues of the three by three matrix (Eq. 17) are given by

$$
\begin{aligned}
\lambda_{1} & =V_{\mathbf{q}}^{z z} \\
\lambda_{2 / 3} & =\frac{1}{2}\left(V_{\mathbf{q}}^{x x}+V_{\mathbf{q}}^{y y} \pm \sqrt{\left(V_{\mathbf{q}}^{x x}-V_{\mathbf{q}}^{y y}\right)^{2}+4\left(V_{\mathbf{q}}^{x y}\right)^{2}}\right),
\end{aligned}
$$

because the off-diagonal components $A_{\mathbf{q}}^{x z}$ and $A_{\mathbf{q}}^{y z}$ vanish for wave vectors in the plane. An out-of-plane orientation of the spins increases the energy $\left(V_{\mathbf{q}}^{z z}\right)$ because of the planar anisotropy. Thus, the wave vector, which maximizes $\lambda_{2}$, describes the classical ground state. In Fig. (1) the exchange energy $J_{\mathbf{q}}$ and the the three components of the symmetrical dipole tensor $A_{\mathbf{q}}^{\alpha \beta}$ are plotted $(a / c=2.39$ and $\left.J^{\prime}=1\right)$. The maximal value for pure antiferromagnetic exchange is found to be for $\mathbf{q}_{0}=4 \pi / 3 a(1,0,0)$, i.e. a $120^{\circ}$ structure.

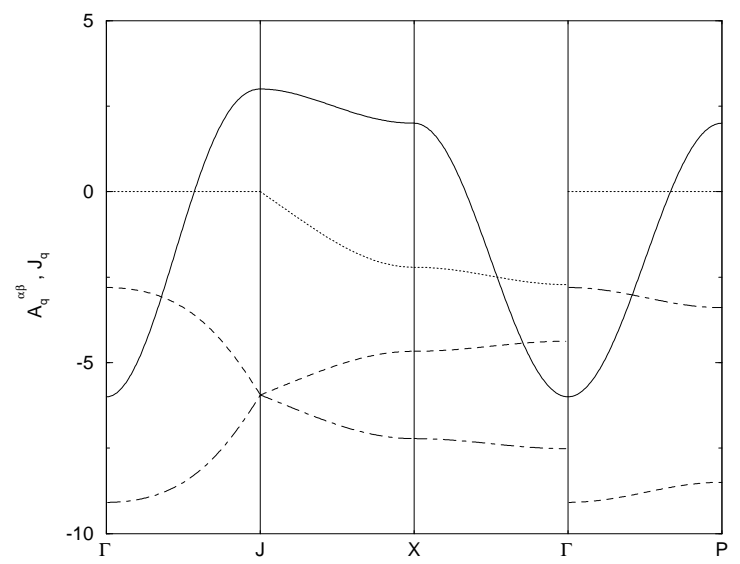

FIG. 1. Antiferromagnetic exchange energy $J_{\mathbf{q}}^{\prime}$ and dipolar interaction $A_{\mathbf{q}}^{\alpha \beta}$ for selected directions in the Brillouin zone. The solid line represents the isotropic exchange, the dashed line $A_{\mathbf{q}}^{y y}$, the dot-dashed line $A_{\mathbf{q}}^{x x}$ and the dotted line $A_{\mathbf{q}}^{x y}$. The dipole components are given in units $\left(g_{L} \mu_{B}\right)^{2} / a_{3}$ and the exchange in units $J^{\prime}$.

For pure dipolar interaction the minimal value is at wave vector $\mathbf{q}=0$, a ferromagnetic orientation in correspondance to the two-dimensional counterpart, the triangular lattice 11 . The ferromagnetic structure is stable as long as the antiferromagnetic exchange energy is lower than the dipolar interaction leading to 


$$
-8 J^{\prime}<A_{0}^{11}-A_{\mathbf{q}_{1}}^{11} \quad \mathbf{q}_{1}=2 \pi / \sqrt{3} a(0,1,0) .
$$

Due to the semiconvergence of the dipole sums for a ferromagnetic structure $(\mathbf{q}=0)$, the dipole components are not analytic at the zone center. For spherically shaped systems the values for the dipole tensor are given in the appendix. For $\mathrm{CsNiF}_{3}$ Eq. (9) reduces to the inequality

$$
J^{\prime}<7 m K \quad \kappa<\kappa_{c 1}=0.20
$$

As a result of the hexagonal symmetry the ferromagnetic ground state is continuously degenerate.

When the exchange energy exceeds the above inequality (Eq. (9)) the classical ground state changes to a collinear antiferromagnetic spin orientation 12. There the spins are orientated along the lattice axes so that there exist only three discrete states called domains (Fig. 5). Domain A can be described by the wave vector $\mathbf{q}_{1}$, domains $\mathrm{C}$ and $\mathrm{B}$ by $\mathbf{q}_{2}=\pi(1,1 / \sqrt{3}, 0)$ and $\mathbf{q}_{3}=\pi(1,-1 / \sqrt{3}, 0)$ respectively. These wave vectors correspond to points $P, X$ and $X^{\prime}$ in the Brillouin zone (Fig. (2)).

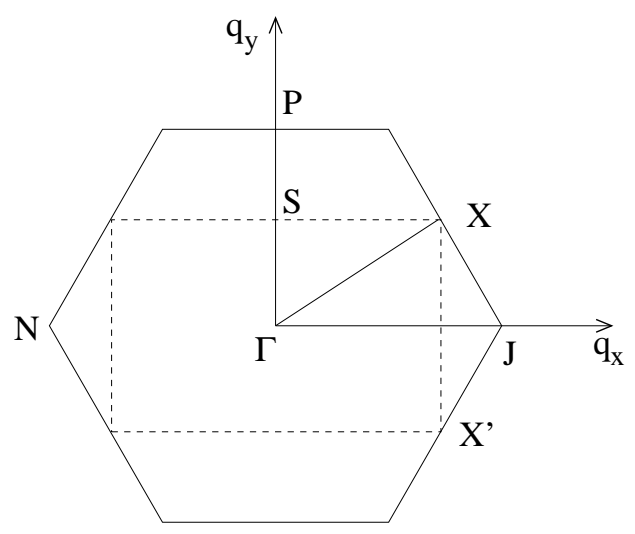

FIG. 2. The Brillouin zones of the hexagonal plane. The hexagon is the crystallographic and the rectangle (dashed) is the magnetic one. Included are the three directions for the scattering measurement which are realized in the domains.

For increasing exchange energy the collinear ground state does not change to a frustrated $120^{\circ}$ structure as one might have assumed. Rather, the system changes to an incommensurate phase. This results from the fact that the dipole energy depends linearly on the wave vector near point $J$, in contrast to a quadratic behavior for the exchange energy (Fig. 1). Therefore, the wave vector for the lowest ground state energy changes continuously from point $J$ towards point $J$, which would correspond to a three sublattice state. Point $J$ is reached only for vanishing dipole energy. Due to the hexagonal symmetry the same situation holds for points $X$ and $X^{\prime}$, i.e. the three-domain structure from the collinear phase survives in the incommensurate region 16 . The three domains and the route to the three sublattice structure can be seen in Fig. (3).

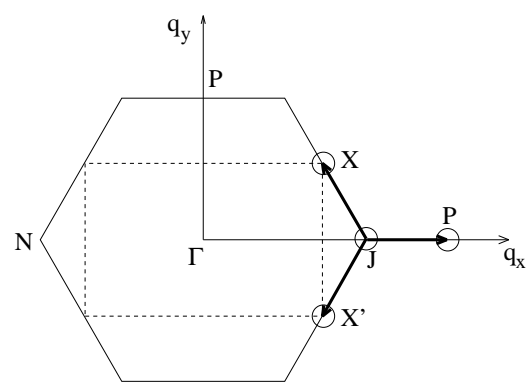

FIG. 3. Transition from the collinear antiferromagnetic phase, points $P, X$ and $X^{\prime}$, towards the three-sublattice phase $J$. The intermediate phases are incommensurate, i.e. the wave vectors, defining the structure, have no commensurate relation to the periodicity of the underlying lattice.

The incommensurate wave vector behaves near $P$ as

$$
\mathbf{q}_{c} \simeq \mathbf{q}_{1}-\sqrt{\frac{\kappa-1.88}{7 \kappa / 24-0.32}}(1,0,0),
$$

i.e. a square root dependence. For increasing exchange energy point $J$ is reached asymptotically (s. Fig. (4)).

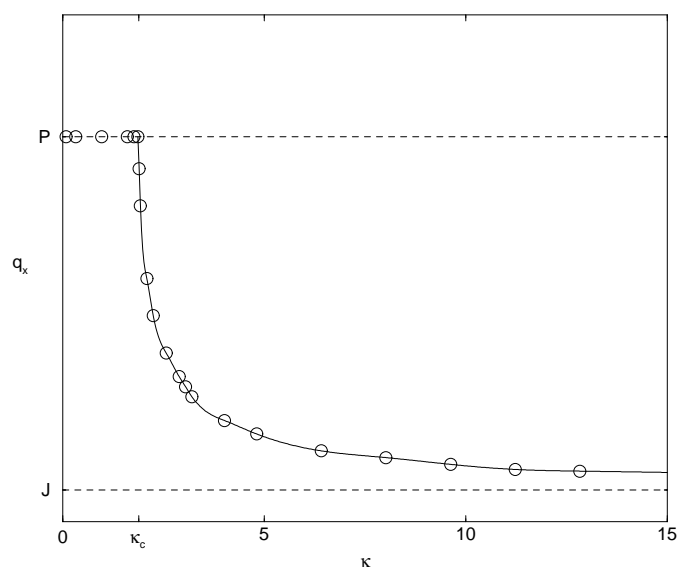

FIG. 4. Incommensurate phase. For $\kappa<\kappa_{c}$ the collinear phase $(P)$ is established, and for larger values the continuous change of the wave vector, describing the intermediate phase, is plotted. The three-sublattice phase $(J)$ is reached only asymptotically. The same route holds for the other two domains.

Near point $J$ the wave vector can be approximated by

$$
\mathbf{q}_{c} \simeq \mathbf{q}_{0}+1.32 \kappa(1,0,0) .
$$

For systems with a dipolar strength as in $\mathrm{CsNiF}_{3}$, the transition to the incommensurate phase yields

$$
J^{\prime}=59 \mathrm{mK} \quad \kappa=\kappa_{c}=1.88 .
$$




\section{EXCITATIONS}

Now we investigate the spin wave frequencies for the two commensurate phases within linear spin wave theory. The incommensurate phase resists such an analysis because of the infinite primitive cell.

In linear spin wave theory the frequencies of the magnons can be derived via the the Holstein-Primakoff transformation (HP) which transforms the spin operators to Bose operators. Therefore, Bose operators $a_{l}$ and $a_{l}^{\dagger} 44$ are introduced, which are given up to bilinear order by

$$
\begin{aligned}
& \tilde{S}_{l}^{z}=\left(S-a_{l}^{\dagger} a_{l}\right), \\
& \tilde{S}_{l}^{x}=\sqrt{\frac{S}{2}}\left(a_{l}+a_{l}^{\dagger}\right), \\
& \tilde{S}_{l}^{y}=-i \sqrt{\frac{S}{2}}\left(a_{l}-a_{l}^{\dagger}\right)
\end{aligned}
$$

with the local spin vector $\tilde{\mathbf{S}}_{l}$. However, first the classical ground state must be determined, and then the the above expressions have to be inserted so that $\tilde{S}_{l}^{z}$ corresponds to the local $z$-component of each spin.

\section{A. Ferromagnetic phase}

In this phase all spins are aligned ferromagnetically in the hexagonal plane. We choose the $z$-component of the spins along the $x$-axis. After HP transformation the Hamilton operator has the form

$$
H=E_{f m}^{c l}+\sum_{\mathbf{q}} A_{\mathbf{q}} a_{\mathbf{q}}^{\dagger} a_{\mathbf{q}}+\frac{1}{2} B_{\mathbf{q}}\left(a_{\mathbf{q}} a_{-\mathbf{q}}+a_{\mathbf{q}}^{\dagger} a_{-\mathbf{q}}^{\dagger}\right)
$$

with the coefficients

$$
\begin{aligned}
A_{\mathbf{q}}= & S A+2 S\left(J_{0}-J_{\mathbf{q}}\right)+2 S\left(J_{0}^{\prime}-J_{\mathbf{q}}^{\prime}\right)+ \\
& S\left(2 A_{0}^{x x}-A_{\mathbf{q}}^{y y}-A_{\mathbf{q}}^{z z}\right) \\
B_{\mathbf{q}}= & -S A+S\left(A_{\mathbf{q}}^{z z}-A_{\mathbf{q}}^{y y}+2 i A_{\mathbf{q}}^{y z}\right)
\end{aligned}
$$

and the classical ground state energy

$$
E_{f m}^{c l}=-N S^{2}\left(J_{0}+J_{0}^{\prime}+A_{0}^{x x}\right) .
$$

The planar anisotropy $A$ does not contribute to this energy. The spin wave frequency following Eq. (15), given by

$$
E_{\mathbf{q}}=\sqrt{A_{\mathbf{q}}^{2}-\left|B_{\mathbf{q}}\right|^{2}}
$$

Due to the rotation symmetry in the plane the magnon frequency vanishes at the zone center. This spectrum becomes unstable for increasing exchange energy $J^{\prime}$ at point $\mathrm{P}$ which corresponds to a transition to the collinear antiferromagnetic phase (s. section III).

\section{B. Collinear antiferromagnetic phase}

In the ground state there exist three domains (Fig. (5)). Spin wave theory is applied to domain A, where the spins are orientated along the $x$-axis. The antiferromagnetic modulation is given by the wave vector $\mathbf{q}_{1}=$ $\frac{2 \pi}{a \sqrt{3}}(0,1,0)$. For domains $\mathrm{C}$ and $\mathrm{B}$ the analogous wave vectors are $\mathbf{q}_{2}=\frac{\pi}{a}(1,1 / \sqrt{3}, 0)$ and $\mathbf{q}_{3}=\frac{\pi}{a}(1,-1 / \sqrt{3}, 0)$ respectively.

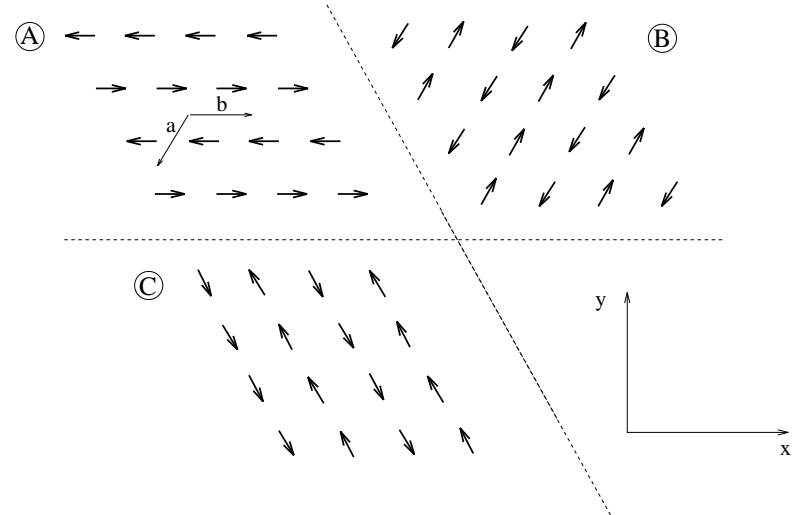

FIG. 5. The ground state for $\mathrm{CsNiF}_{3}$ in the hexagonal plane is one of the three $(\mathrm{A}-\mathrm{C})$ shown configuration called domains. In domain $\mathrm{A}$ the two primitive vectors are represented. The antiferromagnetic modulation can be described by $\mathbf{q}_{1}, \mathbf{q}_{2}$ and $\mathbf{q}_{3}$ for domain A, B and C respectively.

The Holstein-Primakoff transformation can be applied for the crystallographic Brillouin zone 15 by using the factor $e^{i \mathbf{q}_{1} \mathbf{x}_{l}}$ for the antiferromagnetic modulation

$$
\begin{aligned}
H= & E_{g}^{c l}+\sum_{\mathbf{q}}\left\{A_{\mathbf{q}} a_{\mathbf{q}}^{\dagger} a_{\mathbf{q}}+\frac{1}{2} B_{\mathbf{q}}\left(a_{\mathbf{q}} a_{-\mathbf{q}}+a_{\mathbf{q}}^{\dagger} a_{-\mathbf{q}}^{\dagger}\right)+\right. \\
& C_{\mathbf{q}} a_{\mathbf{q}} a_{-\mathbf{q}-\mathbf{q}_{1}}+C_{\mathbf{q}}^{*} a_{\mathbf{q}}^{\dagger} a_{-\mathbf{q}-\mathbf{q}_{1}}^{\dagger}+ \\
& \left.D_{\mathbf{q}} a_{\mathbf{q}}^{\dagger} a_{\mathbf{q}+\mathbf{q}_{1}}+D_{\mathbf{q}}^{*} a_{\mathbf{q}+\mathbf{q}_{1}}^{\dagger} a_{\mathbf{q}}\right\}
\end{aligned}
$$

with the coefficients

$$
\begin{aligned}
A_{\mathbf{q}}= & S\left(2 J_{\mathbf{q}_{1}}-J_{\mathbf{q}}-J_{\mathbf{q}_{\mathbf{q}} \mathbf{q}_{1}}\right)+S A+ \\
& S\left(2 J_{\mathbf{q}_{1}}^{\prime}-J_{\mathbf{q}^{\prime}}^{\prime}-J_{\mathbf{q}_{\mathbf{q} 1}}^{\prime}\right)+S\left(2 A_{\mathbf{q}_{1}}^{x x}-A_{\mathbf{q}_{1}}^{y y}-A_{\mathbf{q}+\mathbf{q}_{1}}^{z z}\right) \\
B_{\mathbf{q}}= & S\left(J_{\mathbf{q}_{\mathbf{q}} \mathbf{q}_{1}}-J_{\mathbf{q}}\right)-S A+ \\
& S\left(J_{\mathbf{q}_{\mathbf{q}}+\mathbf{q}_{1}}^{\prime}-J_{\mathbf{q}}^{\prime}\right)+S\left(A_{\mathbf{q}+\mathbf{q}_{1}}^{z z}-A_{\mathbf{q}}^{y y}\right) \\
C_{\mathbf{q}}= & i S A_{\mathbf{q}}^{y z} \\
D_{\mathbf{q}}= & i S A_{\mathbf{q}}^{y z}
\end{aligned}
$$

and the ground state energy

$$
E_{g}^{c l}=-N S^{2}\left(J_{0}+J_{\mathbf{q}_{1}}^{\prime}+A_{\mathbf{q}_{1}}^{x x}\right) .
$$

Diagonalization of the quadratic Hamiltonian (Eq. (20)) can be done by a generalized Bogoljubov transformation 15 . The magnon frequency is given by 


$$
E_{\mathbf{q}}^{(1 / 2)}=\sqrt{\frac{1}{2}\left(\Omega_{1} \pm \Omega_{2}\right)}
$$

with

$$
\Omega_{1}=A_{\mathbf{q}}^{2}-B_{\mathbf{q}}^{2}+A_{\mathbf{q}+\mathbf{q}_{1}}^{2}-B_{\mathbf{q}+\mathbf{q}_{1}}^{2}+8 C_{\mathbf{q}} C_{\mathbf{q}+\mathbf{q}_{1}}
$$

and

$$
\begin{gathered}
\Omega_{2}^{2}=\left(A_{\mathbf{q}}^{2}-B_{\mathbf{q}}^{2}-A_{\mathbf{q}+\mathbf{q}_{1}}^{2}+B_{\mathbf{q}+\mathbf{q}_{1}}^{2}\right)^{2}+ \\
16\left[C_{\mathbf{q}+\mathbf{q}_{1}}\left(A_{\mathbf{q}+\mathbf{q}_{1}}-B_{\mathbf{q}+\mathbf{q}_{1}}\right)-C_{\mathbf{q}}\left(A_{\mathbf{q}}-B_{\mathbf{q}}\right)\right] \\
\times\left[C_{\mathbf{q}}\left(A_{\mathbf{q}+\mathbf{q}_{1}}+B_{\mathbf{q}+\mathbf{q}_{1}}\right)-C_{\mathbf{q}+\mathbf{q}_{1}}\left(A_{\mathbf{q}}+B_{\mathbf{q}}\right)\right] .
\end{gathered}
$$

Here we changed to the magnetic Brillouin zone, which is half the crystallographic, and therefore two branches appear. Note that the dipole energy lifts the degeneracy for $q_{z} \neq 0$ because of the off-diagonal dipole component $A_{\mathbf{q}}^{y z}$. The splitting of the two branches is of the order of the dipole energy. For wave vectors in the plane the off-diagonal component vanishes and the two magnon branches are degenerate. For wave vectors in the hexagonal plane $\left(q_{z}=0\right)$ stability of the collinear phase requires

$$
A_{\mathbf{q}}>0, \quad A_{\mathbf{q}}>\left|B_{\mathbf{q}}\right|, \quad \mathbf{q}=\left(q_{x}, q_{y}, 0\right) .
$$

From these inequalities we recover the upper bound for the exchange energy $J^{\prime}$ (Eq. (13)). Note that for $J$ and $A$ much larger than $J^{\prime}$ and the dipole energy the inequalities are independent of $J$ and $A$. In Fig. (6) the dispersion relation is plotted 22 for $\mathrm{CsNiF}_{3}$ in two different directions of the Brillouin zone.

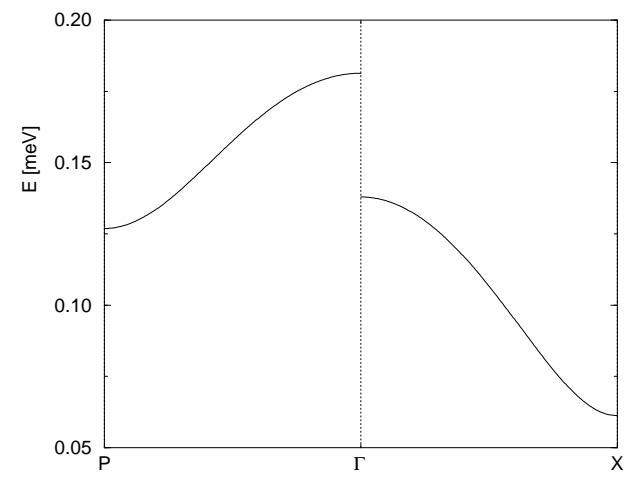

FIG. 6. Dispersion relation for $\mathrm{CsNiF}_{3}$ (vanishing field) for two directions. There is a discontinuity at the zone center.

Note the discontinuity at the zone center, which is a result of the nonanalytic behavior of the dipole-dipole interaction (s. appendix).

\section{COLLINEAR ANTIFERROMAGNETIC PHASE WITH $\mathbf{H}_{0} \neq 0$}

In this section we study the ground state and the dispersion relation for the collinear antiferromagnetic phase in a homogeneous external magnetic field. First we investigate the Néel phase and find that it is stable in the presence of a longitudinal field (parallel to the spin orientation) up to a critical value $H_{0}^{c}$. A transverse field always leads to a reorientation of the spins, i.e. a canted structure. In a second section we study the paramagnetic phase; especially we derive the instability boundary for arbitrary field direction at which the system undergoes a transition to a canted structure. Thus we obtain the corresponding wave vectors characterizing the intermediate phases. In the last section we derive the stability equations for a general two-sublattice structure and the conditions under which conventional spin-flop phases can occur.

\section{A. Néel phase}

We consider domain A with the magnetic field along the $x$-axis. The classical ground state energy (Eq. (24)) is not affected by the magnetic field because of the alternation of the spins. In the bilinear part of the Hamiltonian (Eq. (20)) only the coefficient $D_{\mathbf{q}}$ changes to

$$
D_{\mathbf{q}}=i S A_{\mathbf{q}}^{y z}+\frac{1}{2} g_{L} \mu_{B} H_{0} .
$$

This affects the two branches of the dispersion relation in the following way:

$$
E_{\mathbf{q}}^{(1 / 2)}=\sqrt{\frac{1}{2}\left(\Omega_{1} \pm \Omega_{2}\right)}
$$

with

$\Omega_{1}=A_{\mathbf{q}}^{2}-B_{\mathbf{q}}^{2}+A_{\mathbf{q}+\mathbf{q}_{1}}^{2}-B_{\mathbf{q}+\mathbf{q}_{1}}^{2}+8 C_{\mathbf{q}} C_{\mathbf{q}+\mathbf{q}_{1}}+2\left(g_{L} \mu_{B} H_{0}\right)^{2}$

and

$$
\begin{gathered}
\Omega_{2}^{2}=\left(A_{\mathbf{q}}^{2}-B_{\mathbf{q}}^{2}-A_{\mathbf{q}+\mathbf{q}_{1}}^{2}+B_{\mathbf{q}+\mathbf{q}_{1}}^{2}\right)^{2}+ \\
16\left[C_{\mathbf{q}+\mathbf{q}_{1}}\left(A_{\mathbf{q}+\mathbf{q}_{1}}-B_{\mathbf{q}+\mathbf{q}_{1}}\right)-C_{\mathbf{q}}\left(A_{\mathbf{q}}-B_{\mathbf{q}}\right)\right] \\
\times\left[C_{\mathbf{q}}\left(A_{\mathbf{q}+\mathbf{q}_{1}}+B_{\mathbf{q}+\mathbf{q}_{1}}\right)-C_{\mathbf{q}+\mathbf{q}_{1}}\left(A_{\mathbf{q}}+B_{\mathbf{q}}\right)\right] \\
+4\left(g_{L} \mu_{B} H_{0}\right)^{2}\left(\left(A_{\mathbf{q}}+A_{\mathbf{q}+\mathbf{q}_{1}}\right)^{2}-\left(B_{\mathbf{q}}-B_{\mathbf{q}+\mathbf{q}_{1}}\right)^{2}\right) .
\end{gathered}
$$

The effect of the magnetic field is to lower (lift) the energy of the lower (upper) branch. The collinear ground state is stable as long as the second mode $E_{\mathbf{q}}^{(2)}$ is positive. The energy of this mode vanishes with increasing field strength at first at the boundary of the magnetic 
Brillouin zone at $\mathbf{q}_{4}=\frac{\pi}{a}(1,0,0)$. This implies a critical field of

$$
g_{L} \mu_{B} H_{0}^{c}=2 S \sqrt{\left(A_{\mathbf{q}_{1}}^{x x}-A_{\mathbf{q}_{4}+\mathbf{q}_{1}}^{y y}\right)\left(A_{\mathbf{q}_{1}}^{x x}-A_{\mathbf{q}_{4}}^{y y}\right)} .
$$

Surprisingly, the critical field depends only on the dipolar energy because the antiferromagnetic exchange energy obeys $J_{\mathbf{q}_{4}}^{\prime}=J_{\mathbf{q}_{4}+\mathbf{q}_{1}}^{\prime}=J_{\mathbf{q}_{1}}^{\prime}$. The wave vector $\mathbf{q}_{4}$ describes a four-sublattice system, i.e. the primitive cell of the magnetic lattice consists of four spins. For $\mathrm{CsNiF}_{3} \mathrm{Eq}$. (28) yields for the critical field

$$
H_{0}^{c}=47 \mathrm{mT} .
$$

Above this value the collinear structure becomes unstable and an intermediate state occurs, which is investigated in the next section. The critical value is proportional to the energy gap at wave vector $\mathbf{q}_{4}$ present without field which then is increasingly lowered by applying a field. This phenomenon is already known for systems with an easy-axis anisotropy and a magnetic field parallel to the spinst8.

\section{B. Paramagnetic Phase}

Before studying the noncollinear phase, i.e. the spinflop-like phase, it is instructive to investigate the paramagnetic phase. This phase is established for strong magnetic fields in the hexagonal plane so that all spins align parallel to it. When the magnetic field strength is lowered, the paramagnetic phase becomes unstable and a transition to a canted structure will occcur. From this instability point we obtain the wave vector characterizing the canted structure. Because the ground state of $\mathrm{CsNiF}_{3}$ is not invariant with respect to a rotation around the spin-chain axis (recall that there are three domains AC), the direction of the field plays a crucial role. Thus, we get a non-circular instability line, i.e. the absolute value of the critical field at which the paramagnetic phase becomes unstable depends on the angle the magnetic field encloses with the $x$-axis. In consequence to the hexagonal symmetry it is sufficient to study a range of $60^{\circ}$. In the following $\varphi$ denotes the angle between the external magnetic field $\mathbf{H}_{0}$ and the $x$-axis. After a HP transformation the Hamiltonian reads:

$$
H=E_{P M}^{c l}+\sum_{\mathbf{q}} A_{\mathbf{q}} a_{\mathbf{q}}^{\dagger} a_{\mathbf{q}}+\frac{1}{2}\left(B_{\mathbf{q}} a_{\mathbf{q}} a_{-\mathbf{q}}+B_{\mathbf{q}}^{*} a_{\mathbf{q}}^{\dagger} a_{-\mathbf{q}}^{\dagger}\right)
$$

with the coefficients

$$
\begin{aligned}
A_{\mathbf{q}}= & S\left(2\left(J_{0}-J_{\mathbf{q}}\right)+A+2 J_{0}^{\prime}-2 J_{\mathbf{q}}^{\prime}\right)+g_{L} \mu_{B} H_{0} \\
& +S\left(2 A_{0}^{x x}-A_{\mathbf{q}}^{z z}-\sin ^{2} \varphi A_{\mathbf{q}}^{x x}-\cos ^{2} \varphi A_{\mathbf{q}}^{y y}\right. \\
& \left.-\sin 2 \varphi A_{\mathbf{q}}^{x y}\right) \\
B_{\mathbf{q}}= & S\left(-A+A_{\mathbf{q}}^{z z}-\sin ^{2} \varphi A_{\mathbf{q}}^{x x}-\cos ^{2} \varphi A_{\mathbf{q}}^{y y}+\sin 2 \varphi A_{\mathbf{q}}^{x y}\right. \\
& \left.+2 i \cos \varphi A_{\mathbf{q}}^{y z}-2 i \sin \varphi A_{\mathbf{q}}^{x z}\right),
\end{aligned}
$$

and the classical ground state energy of the paramagnetic state

$$
E_{P M}^{c l}=-N S^{2}\left(J_{0}+J_{0}^{\prime}+A_{0}^{x x}\right)-g_{L} \mu_{B} N S H_{0},
$$

which is independent of the direction of the field. The dispersion relation for the paramagnetic phase is given by

$$
E_{\mathbf{q}}=\sqrt{A_{\mathbf{q}}^{2}-\left|B_{\mathbf{q}}\right|^{2}}
$$

In Fig. (7) the excitation frequencies are shown for different field directions $(\varphi=0)$.
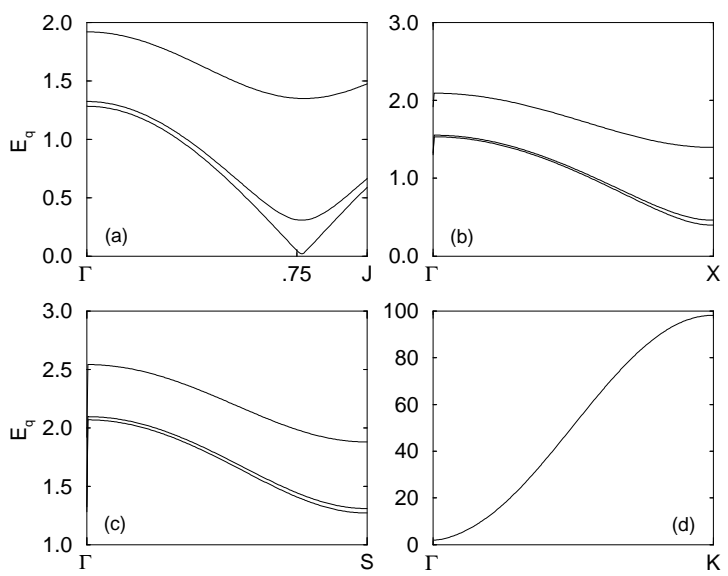

FIG. 7. Dispersion relation for $\mathrm{CsNiF}_{3}$ in the paramagnetic phase. The field is aligned along the $x$-axis $(\varphi=0)$. The upper curves are calculated for $H_{0}=2.1 H_{0}^{c x}$, the middle curves for $H_{0}=1.06 H_{0}^{c x}$ and the lower curves are just above the critical field $H_{0}^{c x}$. Point $\mathrm{K}$ equals $\mathbf{q}=\frac{\pi}{c}(0,0,1)$. In graph (a) one can see the softening of the dispersion at an incommensurate wave vector.

When the field is lowered the paramagnetic phase becomes unstable below a critical field, given by the zero of the excitation energy $\left(q_{z}=0, A_{\mathbf{q}}^{y z}=0\right)$ :

$$
\begin{aligned}
g_{L} \mu_{B} H_{0}^{c}= & 2 S\left(J_{\mathbf{q}}^{\prime}-J_{0}^{\prime}-A_{0}^{x x}+\sin ^{2} \varphi A_{\mathbf{q}}^{x x}+\cos ^{2} \varphi A_{\mathbf{q}}^{y y}\right. \\
& \left.-\sin 2 \varphi A_{\mathbf{q}}^{x y}\right) .
\end{aligned}
$$

Considered as a function of $\mathbf{q}$ the maximum of this expression gives the critical value (dependent on the angle), and the wave vector $\mathbf{q}$ reflects the ordering of the phase below. Assuming a conventional spin-flop phase or an intermediate phase (two sublattice structure) we would expect the maximum value for the critical field at wave vectors describing the antiferromagnetic domains, i.e. $\mathbf{q}_{1}, \mathbf{q}_{2}$ or $\mathbf{q}_{3}$. However, the detailed analysis showed that the paramagnetic phase gets unstable at an incommensurate wave vector $\tilde{\mathbf{q}}(\varphi)$ for certain field directions. Before considering the general case we study the special angles $\varphi=0^{\circ}$ and $\varphi=90^{\circ}$. 

to:

a. $\varphi=0^{\circ}$ : For this field direction Eq. (35) simplifies

$$
g_{L} \mu_{B} H_{0}^{c x}=2 S\left(J_{\mathbf{q}}^{\prime}-J_{0}^{\prime}-A_{0}^{x x}+A_{\mathbf{q}}^{y y}\right) .
$$

This expression is proportional to $V_{\mathbf{q}}^{y y}$ defined in Eq. (7). The maximum of this component is not achieved for $\mathbf{q}_{1}$ but around $\mathbf{q}_{0}$. In the limit of vanishing dipole energy the incommensurate wave vector is given by (compare with Eq. (12))

$$
\tilde{\mathbf{q}}\left(0^{\circ}\right) \simeq \mathbf{q}_{0}-1.32 \kappa(1,0,0),
$$

i.e. the phase below the paramagnetic phase is an incommensurate phase resembling a three sublattice structure. Note that this incommensurate wave vector is different from the wave vector found for the case of vanishing dipole energy (note the minus sig in Eq. (12)). For increasing dipole energy the wave vector (Eq. (37)) changes continuously to $\mathbf{q}=\mathbf{0}$, implying that for $\kappa \rightarrow 0$ the system orders ferromagnetically (s. III).

For $\mathrm{CsNiF}_{3}$ the critical magnetic field can be evaluated to

$$
H_{0}^{c x}=290 \mathrm{mT} \quad \text { at } \quad \tilde{\mathbf{q}}(0)=\frac{\pi}{a}(1.023,0,0) .
$$

The softening of this mode can be seen in Fig. (ㄱ(a)). This incommensurate wave vector happens to be near the wave vector $\mathbf{q}_{4}$, the four-sublattice structure, which describes an antiferromagnetic modulation along the $x$ axis. Note that $\mathbf{q}_{4}$ is the corresponding wave vector at which the Néel state becomes unstable (compare section A).

b. $\varphi=90^{\circ}$ : For magnetic fields parallel to the $y$-axis the critical value is given by

$$
g_{L} \mu_{B} H_{0}^{c y}=2 S\left(J_{\mathbf{q}}^{\prime}-J_{0}^{\prime}-A_{0}^{x x}+A_{\mathbf{q}}^{x x}\right) .
$$

This expression is found to have its maximum value at $\mathbf{q}_{1}$, the antiferromagnetic wave vector of the collinear phase of domain A. For $\mathrm{CsNiF}_{3}$ this yields

$$
H_{0}^{c y}=340 \mathrm{mT} \quad \text { at } \quad \tilde{\mathbf{q}}\left(90^{\circ}\right)=\mathbf{q}_{1} .
$$

At $H_{0}^{c y}$ the system undergoes a transition into a commensurate phase, precisely the two sublattice phase corresponding to $\mathbf{q}_{1}$. When the antiferromagnetic exchange energy $J^{\prime}$ is lowered, the critical field vanishes for $\kappa \leq$ 0.20 . This limiting value agrees with the value for the transition between the ferromagnetic and the collinear antiferromagnetic ground state (Eq. (10)). In contrast to the previous case $\left(\varphi=0^{\circ}\right)$, for this field direction there does not exist a region for $\kappa$ where an intermediate phase between ferromagnetic and paramagnetic phase occurs. Owing to the hexagonal symmetry the critical field for $\varphi=30^{\circ}$ is the same as for $\varphi=90^{\circ}\left(\varphi=-30^{\circ}\right)$ but at the antiferromagnetic wave vector $\mathbf{q}_{2}\left(\mathbf{q}_{3}\right)$ characterizing domain $\mathrm{C}(\mathrm{B})$. c. $\varphi=$ arbitrary: Finally we turn to arbitrary angles, for which the situation turns out to be nontrivial. The complete dependence of the field direction $\tilde{\mathbf{q}}(\varphi)$ for $\mathrm{CsNiF}_{3}$ is plotted in Fig. 8.

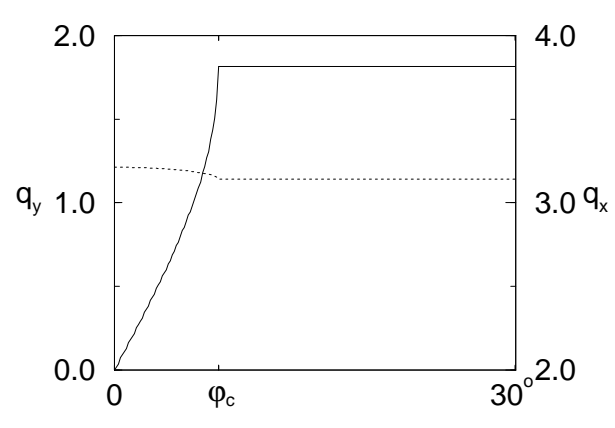

FIG. 8. Wave vector $\tilde{q}_{y}$ and $\tilde{q}_{x}$ in the paramagnetic phase at which the phase gets unstable depending on the direction of the external magnetic field. We used the parameters for $\mathrm{CsNiF}_{3} . \varphi$ measures the angle between the magnetic field and the $x$-axis. For $\varphi>7.8^{\circ}$ the instability appears at point $\mathrm{X}$.

The wave vector components $\tilde{q}_{x}$ and $\tilde{q}_{y}$ are plotted as a function of the angle $\varphi$ for the region in question. A critical angle $\varphi_{c} \approx 7.8^{\circ}$ is observed above which the wave vector is locked to $\mathbf{q}_{2}$. Varying the angle $\varphi$ between $0^{\circ}$ and $7.8^{\circ}$ the wave vector where the instability sets in changes continuously from $\tilde{\mathbf{q}}(0)$ to $\tilde{\mathbf{q}}\left(\varphi_{c}\right)=\mathbf{q}_{2}$. In Fig. (9) the critical value for the magnetic field is plotted as a function of the angle for the same angular domain.

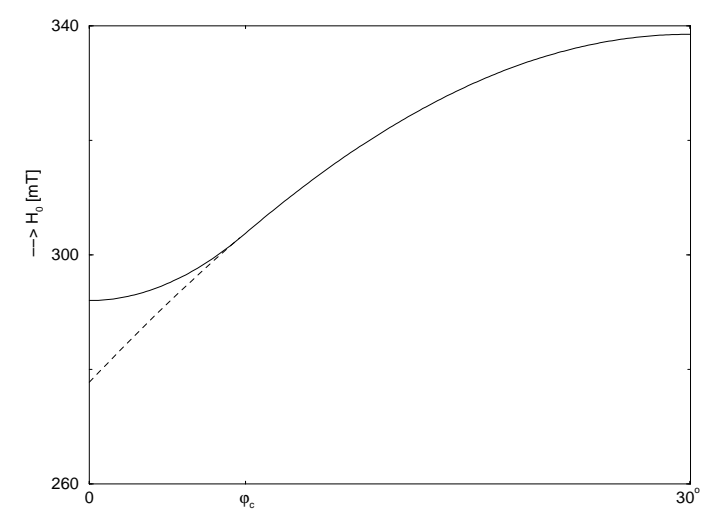


FIG. 9. Critical field below which the paramagnetic phase gets unstable for field direction between $0^{\circ}$ and $30^{\circ}$. Note that there is no discontinuity at $\varphi_{c}=7.8^{\circ}$ but a slight kink. For smaller (larger) angles the paramagnetic phase changes to an incommensurate (commensurate) phase. The dashed curve indicates the critical field when assuming that the soft mode occurs at $\mathbf{q}_{2}$.

Note that the critical field is a continuous curve even at the critical angle $\varphi_{c}$. It has only a small kink at this point. The dashed curve results from Eq. (35) when it is assumed that the instability point occurs at $\mathbf{q}_{2}$ for the whole angular segment. Thus we can see that for $\varphi<\varphi_{c}$ the incommensurate structure is favored. Due to the inversion symmetry of the lattice together with $\tilde{\mathbf{q}}(\varphi)$ there is a second modulation wave vector $-\tilde{\mathbf{q}}(\varphi)$. The result of this investigation for a complete rotation of the magnetic field is summarized in Fig. (10): The instability line of the paramagnetic phase is shown. There are angular regions where the paramagnetic structure undergoes a transition to a two-sublattice structure (annotated by the corresponding wave vector) separated by regions with incommensurate structures which are plotted with a thick line. The three dashed lines around zero indicate the Néel phases for a longitudinal field.

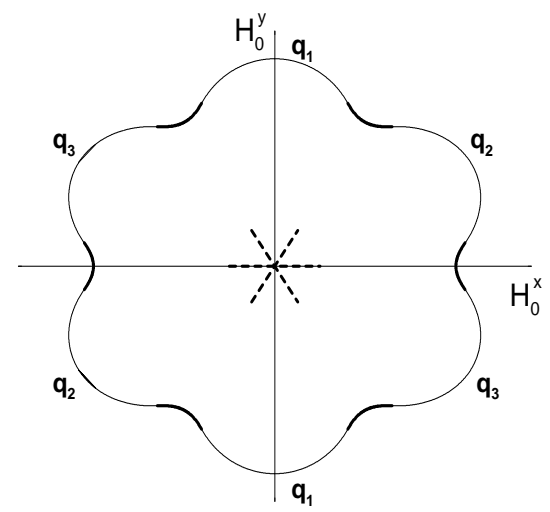

FIG. 10. Angular dependence of the instability of the paramagnetic phase for $\mathrm{CsNiF}_{3}$-like systems. The thick-lined segments on the instability curve correspond to the direction of the magnetic field $\mathbf{H}_{0}=\mathrm{H}_{0}(\cos \varphi, \sin \varphi, 0)$, at which the paramagnetic phase changes to an incommensurate phase. They are seperated by segments where a transition to a commensurate structure happens, especially a two-sublattice structure. The corresponding wave vector is given. The dashed lines denote the Néel state.

In Fig. (11) the full wave vector dependence within the Brillouin zone is shown for $\mathrm{CsNiF}_{3}$. Note that the wave vector is locked at the two-sublattice wave vectors, which cannot be seen in this representation.

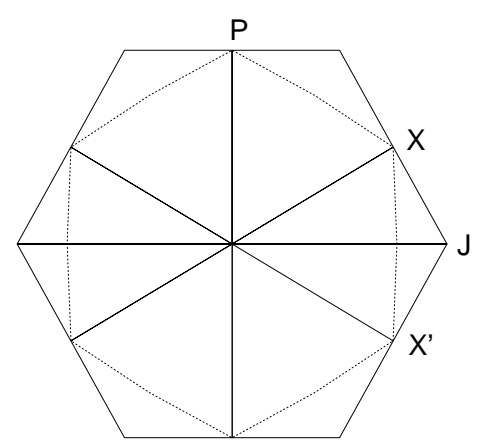

FIG. 11. Angular dependence of the wave vector $\tilde{\mathbf{q}}(\varphi)$ on the instability curve from Fig. (10) for $\mathrm{CsNiF}_{3}$. Shown is the Brillouin zone from Fig. (6). The wave vector happens to be near the edge of the magnetic Brillouin zone for the special value of $\kappa=0.79$.

Now we want to derive the critical angle $\varphi_{c}$, at which the commensurate and the incommensurate phases coexist, for different strengths of the antiferromagnetic exchange energy $J^{\prime}$. This value can be obtained by evaluation of the maximum of Eq. (35) near $\mathbf{q}_{1}$. Expanding the exchange energy and the dipole energy in a Taylor series we get

$$
\begin{aligned}
& J_{\tilde{\mathbf{q}}+\mathbf{q}_{1}}^{\prime} \approx J_{\mathbf{q}_{1}}+\frac{1}{2} J^{\prime}\left(\tilde{q}_{x}^{2}-3 \tilde{q}_{y}^{2}\right) \\
& A_{\tilde{\mathbf{q}}+\mathbf{q}_{1}}^{x x} \approx A_{\mathbf{q}_{1}}^{x x}-G^{\prime}\left(0.816 \tilde{q}_{x}^{2}+0.112 \tilde{q}_{y}^{2}\right) \\
& A_{\tilde{\mathbf{q}}+\mathbf{q}_{1}}^{y y} \approx A_{\mathbf{q}_{1}}^{y y}+G^{\prime}\left(0.816 \tilde{q}_{x}^{2}-0.112 \tilde{q}_{y}^{2}\right) \\
& A_{\tilde{\mathbf{q}}+\mathbf{q}_{1}}^{x y} \approx G^{\prime} 1.361 \tilde{q}_{x} \tilde{q}_{y},
\end{aligned}
$$

where we introduced the dipolar strength $G^{\prime}=G / a_{3}$ (s. appendix). Inserting in Eq. (35) and setting the determinant of the Hess matrix zero we find the critical angle as a function of the exchange energy $J^{\prime}$ which is shown in Fig. (12).

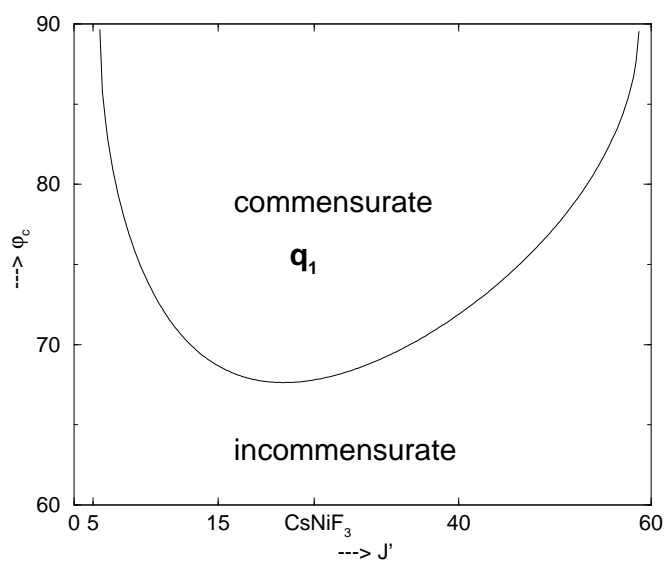


FIG. 12. The dependence of the critical angle $\varphi_{c}$ on the angtiferromagnetic exchange energy $J^{\prime}$. The region between the curve and $\varphi=90^{\circ}$ denotes the commensurate structure right below the instability curve.

One can see that the angular segments of the commensurate structure decrease when the antiferromagnetic exchange energy takes values near the upper or the lower bound of the collinear antiferromagnetic phase. For $\mathrm{CsNiF}_{3}$ it happens that the commensurate segments have nearly the largest possible size.

\section{Spin-flop phase, intermediate phase}

In this section we want to examine the noncollinear phase, i.e. the region within the instability curve of Fig. (10). In the following we investigate a magnetic field along the $x$-axis. Concerning domains $\mathrm{B}$ and $\mathrm{C}$ it is clear that for finite field strength the spins reorientate in order to gain energy from the interaction with the magnetic field, while domain A remaining in a Néel state cannot gain Zeeman energy. Thus the Néel state cannot be a stable state. In section A we showed via stability calculations that a Néel structure is stabilized up to a critical value (Eq. (28)). Thus the Néel state for domain A cannot be a stable state but must be metastable. There is an activation energy necessary to flip domain $\mathrm{A}$ in a ground state emerging for domains $\mathrm{B}$ or $\mathrm{C}$ in the presence of a field, due to the broken rotation symmetry in the hexagonal plane. The situation for a virgin probe is as follows: without magnetic field the system might be built up of the three domains with equal volume. Raising the magnetic field (parallel to the spins in domain A) does not change the spin orientation in domain $\mathrm{A}$ but leads to a slight reorientation in domains $\mathrm{B}$ and $\mathrm{C}$. Above the the critical value (Eq. (28)) the spins in domain A flip (first order phase transition) to an orientation identical to either domain B or domain C. For strong magnetic fields one finally enters the paramagnetic phase. When the magnetic field is decreased thereafter the spins order again in the two domains, but domain $\mathrm{A}$ is never formed again because of the metastability of this domain with respect to fields along the $x$-direction. The system ends up in a state where only domains $\mathrm{B}$ and $\mathrm{C}$ are present. In this scenario we neglected thermal effects, possible domain wall energies and defects in the crystal which should be taken into account for a proper description. In fact, the experiments do not show this scenario; the system alyays ends in a three-domain state after a magnetic cycle?.

For general field direction we expect from the investigation of the instability line of the paramagnetic phase that in the intermediate phase there are angular regions with incommensurate modulations separated by region with a commensurate two-sublattice modulation. Here we want to find out the conditions for existence of a conventional spin-flop phase, i.e. the ground state consists of two sublattices on each of which the spin component along the field is the same $(\alpha=\beta$ in Fig. (13)).

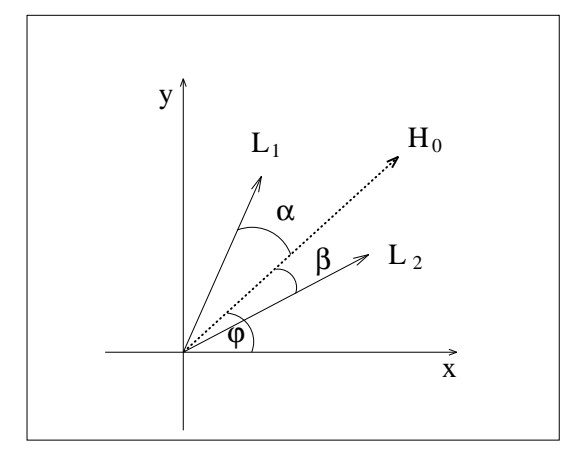

FIG. 13. Coordinate system for a general two-sublattice spin orientation. The spins of sublattice $\mathcal{L}_{1}\left(\mathcal{L}_{2}\right)$ enclose an angle of $\alpha(\beta)$ with the magnetic field $\mathbf{H}_{0}=H_{0}(\cos \varphi, \sin \varphi, 0)$.

Due to the large planar anisotropy $A$ the spins reorientate only within the plane, i.e. we need only one angle relative to the magnetic field for each spin. A two sublattice structure is characterized by the wave vectors $\mathbf{q}_{1}$, $\mathbf{q}_{2}$ and $\mathbf{q}_{3}$. Introducing (Fig. (13)) the angle enclosing the $x$-axis and the magnetic field $\mathbf{H}_{0}$ by $\varphi$ and the angle enclosing the field and the orientation of the spin on the first (second) sublattice $\mathcal{L}_{1}\left(\mathcal{L}_{2}\right)$ by $\alpha(\beta)$, the ground state energy can be written in the form:

$$
\begin{aligned}
E_{I N}^{c l}= & -N S^{2}\left\{J_{0}+\cos ^{2} \gamma\left[J_{0}^{\prime}+A_{0}^{x x}\right]+\right. \\
& \sin ^{2} \gamma\left[J_{\mathbf{q}}^{\prime}+\sin ^{2}(\varphi+\delta) A_{\mathbf{q}}^{x x}+\cos ^{2}(\varphi+\delta) A_{\mathbf{q}}^{y y}\right. \\
& \left.\left.+\sin 2(\varphi+\delta) A_{\mathbf{q}}^{x y}\right]\right\}-g_{L} \mu_{B} N S H_{0} \cos \gamma \cos \delta,
\end{aligned}
$$

where $\mathbf{q}$ denotes one of the above mentioned twosublattice wave vectors. Here we used the more appropriate variables $\gamma$ and $\delta$, which are related to the original angles by

$$
\gamma=\frac{\alpha+\beta}{2}, \quad \delta=\frac{\alpha-\beta}{2}
$$

The minimum value of the ground state energy requires $\left(\partial_{\gamma} E_{I N}^{c l}=0\right.$ and $\left.\partial_{\delta} E_{I N}^{c l}=0\right)$ :

$$
\begin{array}{r}
g_{L} \mu_{B} H_{0} \cos \delta=2 S\left[J_{\mathbf{q}}^{\prime}-J_{0}^{\prime}-A_{0}^{x x}+\sin ^{2}(\varphi+\delta) A_{\mathbf{q}}^{x x}\right. \\
\left.+\cos ^{2}(\varphi+\delta) A_{\mathbf{q}}^{y y}+\sin 2(\varphi+\delta) A_{\mathbf{q}}^{x y}\right] \cos \gamma \\
g_{L} \mu_{B} H_{0} \cos \gamma \sin \delta=S \sin ^{2} \gamma\left[\sin 2(\varphi+\delta)\left(A_{\mathbf{q}}^{x x}-A_{\mathbf{q}}^{y y}\right)\right. \\
\left.+2 \cos 2(\varphi+\delta) A_{\mathbf{q}}^{x y}\right] .
\end{array}
$$

These equations will be discussed now for a spin-flop and an intermediate spin structure.

\section{Spin-flop phase}

For a conventional spin-flop phase $(\alpha=\beta=\gamma$ and $\delta=0)$ these equations can be simplified 


$$
\begin{aligned}
g_{L} \mu_{B} H_{0}= & 2 S\left[J_{\mathbf{q}}^{\prime}-J_{0}^{\prime}-A_{0}^{x x}+\sin ^{2} \varphi A_{\mathbf{q}}^{x x}+\cos ^{2} \varphi A_{\mathbf{q}}^{y y}\right. \\
& \left.+\sin 2 \varphi A_{\mathbf{q}}^{x y}\right] \cos \gamma \\
0= & \sin 2 \varphi\left(A_{\mathbf{q}}^{x x}-A_{\mathbf{q}}^{y y}\right)+2 \cos 2 \varphi A_{\mathbf{q}}^{x y}
\end{aligned}
$$

The second relation gives a condition for the existence of a conventional spin-flop phase. By using the values of the dipole energies and the dipole relations given in the appendix we find the following solutions for Eq. (49):

\begin{tabular}{|c|ccc|}
\hline$\varphi$ & $\mathbf{q}_{1}$ & $\mathbf{q}_{2}$ & $\mathbf{q}_{3}$ \\
\hline \hline stable & $\frac{\pi}{2}, \frac{3 \pi}{2}$ & $\frac{\pi}{6}, \frac{7 \pi}{6}$ & $\frac{5 \pi}{6}, \frac{11 \pi}{6}$ \\
unstable & $0, \pi$ & $\frac{2 \pi}{3}, \frac{5 \pi}{3}$ & $\frac{\pi}{3}, \frac{4 \pi}{3}$ \\
\hline
\end{tabular}

In the second row the angles are given for the corresponding wave vector. These solutions represent magnetic field directions perpendicular to the spin orientation for vanishing field. In the third row we tabulated the solutions which correspond to field directions along the spin orientation. The latter solutions represent no minimal ground state energy for exchange energies $J^{\prime}<26 \mathrm{~m} \mathrm{~K}$. This can be seen from Eq. (48), where the term in brackets then is negative. Even for $H_{0}=0$ and e.g. $\varphi=0^{\circ}$ the ground state energy

$$
E_{I N}^{g}=-N S^{2}\left(J_{0}+J_{\mathbf{q}_{1}}^{\prime}+A_{\mathbf{q}_{1}}^{y y}\right)
$$

is larger than the ground state energy for the Néel state (Eq. (24)). Thus, there is no spin-flop phase for longitudinal fields in $\mathrm{CsNiF}_{3}$.

Inserting the values (stable) for the angles from the table in Eq. (48) we get the static equilibrium relation between the magnetic field and the angle $\gamma$

$$
g_{L} \mu_{B} H_{0}=2 S\left[J_{\mathbf{q}}^{\prime}-J_{0}^{\prime}-A_{0}^{x x}+A_{\mathbf{q}_{1}}^{x x}\right] \cos \gamma .
$$

Note that this relation holds for all stability values of $\varphi$ due to the hexagonal symmetry, as can be seen by applying the dipole relations from the appendix. So we conclude that for most angles $\varphi$ there does not exist a spin-flop phase except for the above found solutions.

\section{Intermediate phase}

In the general case of two independent angles we have to solve equations (48) and (49). As a result one obtains the dependence of the angles $\alpha\left(H_{0}, \varphi\right)$ and $\beta\left(H_{0}, \varphi\right)$ upon the field strength and the direction. Since the solution for the general case is nontrivial we want to study only two special situations, namely $\varphi=0, \varphi=90^{\circ}$ and a modulation wave vector $\mathbf{q}_{1}$ for domain A. In these two cases the equations of stability (Eqs. (48) and (49)) can be solved analytically. For $\varphi=90^{\circ}$ we find that the intermediate phase has a higher ground state energy than the conventional spin-flop phase $(\alpha=\beta)$. For $\varphi=0$ the ground state energy of the intermediate phase exceeds the energy for the Néel state for vanishing fields, signifying the absence of such a state. The complete investigation of the general case is left for future studies.

\section{SUMMARY}

We have studied the ground state for a planar, quasi one-dimensional ferromagnet with weak antiferromagnetic exchange and dipole-dipole interaction. For vanishing exchange energy the spins order ferromagnetically in the hexagonal plane. With increasing exchange energy the ground state passes through a collinear phase with three possible domains and switches over to an incommensurate phase, where the characterizing wave vector moves continuously from the points $P, X$ or $X^{\prime}$ to the three-sublattice wave vector at point $J$ (Fig. (3)). The three-domain structure of the collinear phase persists in the incommensurate phase.

Via linear spin wave theory we obtain the dispersion relation for the ferromagnetic and the collinear antiferromagnetic phase $\left(H_{0}=0\right)$. The range for the collinear antiferromagnetic phase for $\mathrm{CsNiF}_{3}$ like systems is calculated from ground state energy calculations and from stability conditions concerning to excitations

$$
7 \mathrm{mK}<J^{\prime}<59 \mathrm{mK}
$$

which is consistent with $J^{\prime}=25 \mathrm{mK}$ found in recent measurements12. The upper and lower bounds are independent of the ferromagnetic exchange energy $J$ and the planar anisotropy energy $A$, as long as they are much larger than the dipole energy and the exchange energy $J^{\prime}$.

In the following we studied the magnetic phase diagram of the collinear antiferromagnetic phase. For homogeneous external magnetic fields parallel to the spin orientation the Néel phase is stable up to a critical field which depends only on the dipolar energy (Eq. (28)). The instability is shown by the softening of a mode with wave vector $\mathbf{q}_{4}$ which describes a transition to a foursublattice structure. For magnetic fields transverse to the spins a reorientation sets in for all finite field values.

By stability investigations of the paramagnetic phase we obtained a non-circular instability line for fields in the hexagonal plane. There is a strong dependence on the field direction on the critical field and the structure of the phase below the paramagnetic phase. For certain angular domains the system changes in incommensurate structures which are separated by commensurate (twosublattice) structures (Fig. (10)). We showed that the commensurate angular domains are intermediate phases while conventional spin-flop phases exist only for magnetic field directions $\varphi=30^{\circ} \neq n 60^{\circ}$. This is in contrast to the result of Yamazaki et al.16, who found conventional spin-flop phases for all field directions, even for a longitudinal field where an incommensurate phase should set in from our calculations. This may result from their semiclassical model which does not consider the full nature of the dipole-dipole interaction.

As a result, the magnetic phase diagram reveals an interesting structure of different phases. The investigation 
of the complete magnetic phase diagram (intermediate phase) is left for future studies.

\section{ACKNOWLEDGMENTS}

This work has been supported by the German Federal Ministry for Research and Technology (BMBF) under the contract number 03-SC4TUM. The work of C.P. has been supported by the Deutsche Forschungsgemeinschaft (DFG) under the contract no. PI 337/1-1.

\section{DIPOLE-DIPOLE INTERACTION}

In this section we summarize the most important relations concerning the dipole-dipole interaction needed in the analysis. We consider a simple hexagonal lattice, with lattice constant $a$ in the triangular plane and lattice constant $c$ perpendicular to the plane. The direct lattice is paramatrized by

$$
\begin{gathered}
\mathbf{x}_{l}=a\left(l_{1}, 0,0\right)+\frac{a}{2}\left(l_{1}, \sqrt{3} l_{2}, 0\right)+c\left(0,0, l_{3}\right), \\
l_{i}=0, \pm 1, \pm 2, \ldots
\end{gathered}
$$

and the reciprocal lattice by

$$
\begin{gathered}
\mathbf{G}=\frac{2 \pi}{a}\left(h_{1}, 0,0\right)+\frac{2 \pi}{\sqrt{3} a}\left(2 h_{2}-h_{1}, 0\right)+\frac{2 \pi}{c}\left(0,0, h_{3}\right), \\
h_{i}=0, \pm 1, \pm 2, \ldots
\end{gathered}
$$

The Fourier transform of the dipole energy (Eq. (21)

$$
\begin{gathered}
A_{\mathbf{q}}^{\alpha \beta}= \\
-G \lim _{\mathbf{x} \rightarrow 0} \frac{1}{2}\left(\sum_{l} e^{i \mathbf{q} \mathbf{x}_{l}}\left(\frac{\delta^{\alpha \beta}}{\left|\mathbf{x}-\mathbf{x}_{l}\right|^{3}}-\frac{3 x_{l}^{\alpha} x_{l}^{\beta}}{\left|\mathbf{x}-\mathbf{x}_{l}\right|^{5}}\right)-\frac{1}{|\mathbf{x}|^{3}}\right)
\end{gathered}
$$

with

$$
G=\left(g_{L} \mu_{B}\right)^{2}
$$

is found by the Ewald summation technique 13 . There the summation is split into a part over the direct lattice and a part over the reciprocal lattice and, by using in an intermediate step the generalized theta function 13 , we derive the following relation $\left(a_{3}=\frac{\sqrt{3}}{2} a^{2} c\right)$ :

$$
\begin{aligned}
A_{\mathbf{q}}^{\alpha \beta} \frac{a_{3}}{G}= & \frac{2 \pi}{3} \delta^{\alpha \beta}-\pi \delta^{\alpha \beta} \sum_{l}^{\prime} e^{i \mathbf{q} \mathbf{x}_{l}} \varphi_{1 / 2}\left(\frac{\pi}{a_{3}^{2 / 3}}\left|\mathbf{x}_{l}\right|^{2}\right) \\
& +\frac{2 \pi^{2}}{a_{3}^{2 / 3}} \sum_{l}^{\prime} e^{i \mathbf{q} \mathbf{x}_{l}} x_{l}^{\alpha} x_{l}^{\beta} \varphi_{3 / 2}\left(\frac{\pi}{a_{3}^{2 / 3}}\left|\mathbf{x}_{l}\right|^{2}\right)
\end{aligned}
$$

$$
-\frac{a_{3}^{2 / 3}}{2} \sum_{\mathbf{G}}(\mathbf{q}+\mathbf{G})^{\alpha}(\mathbf{q}+\mathbf{G})^{\beta} \varphi_{0}\left(\frac{a_{3}^{2 / 3}}{4 \pi}|\mathbf{q}+\mathbf{G}|^{2}\right) .
$$

Here $l$ refers to the direct lattice and $\mathbf{G}$ to the reciprocal lattice. The prime excludes the value $l=0$ and we used the Misra functions

$$
\varphi_{n}(x)=\int_{1}^{\infty} d t t^{n} e^{-x t}
$$

Expanding the dipole tensor for small wave vectors yields:

$$
\begin{aligned}
A_{\mathbf{q}}^{\alpha \beta} \frac{a_{3}}{G}= & -2 \pi\left(\frac{q^{\alpha} q^{\beta}}{q^{2}}-\frac{\delta^{\alpha \beta}}{3}\right)-\pi \delta^{\alpha \beta} \sum_{l}^{\prime} \varphi_{1 / 2}\left(\frac{\pi}{a_{3}^{2 / 3}}\left|\mathbf{x}_{l}\right|^{2}\right) \\
& +\frac{2 \pi^{2}}{a_{3}^{2 / 3}} \sum_{l}^{\prime} x_{l}^{\alpha} x_{l}^{\beta} \varphi_{3 / 2}\left(\frac{\pi}{a_{3}^{2 / 3}}\left|\mathbf{x}_{l}\right|^{2}\right) \\
& +\sum_{\mathbf{G}}^{\prime} G^{\alpha} G^{\beta} \varphi_{0}\left(\frac{a_{3}^{2 / 3}|\mathbf{G}|^{2}}{4 \pi}\right)+\mathcal{O}\left(q^{\alpha} q^{\beta}\right) \\
= & -2 \pi\left(\frac{q^{\alpha} q^{\beta}}{q^{2}}-\frac{\delta^{\alpha \beta}}{3}\right)+a_{\text {lat }},
\end{aligned}
$$

where we have introduced a lattice dependent term $a_{\text {lat }}$. This expression is non-analytic due to the semiconvergence of the dipole sum in three dimensions. The value at $\mathbf{q}=0$ depends on the direction of the limiting process expressing the shape dependence of the system. For a cubic lattice the lattice dependent term vanishes $a_{\text {lat }}=0$, so the dipole energy for a ferromagnetic structure is zero. In contrast, this term does not vanish for a hexagonal lattice. For non-spherical surfaces this equation has to be corrected by the demagnetization factor.

In the following table the dipole components are listed for selected wave vectors in units of $G / a_{3}$ and for $k=$ $a / c=2.39$, the ratio of the lattice constants for $\mathrm{CsNiF}$ $\left(q_{z}=0\right)$

\begin{tabular}{|c|cccccc|}
\hline & $\mathbf{q}=0$ & $\mathbf{q}_{0}$ & $\mathbf{q}_{1}$ & $\mathbf{q}_{2}$ & $\mathbf{q}_{3}$ & $\mathbf{q}_{4}$ \\
\hline$A_{\mathbf{q}}^{x x}$ & -2.805 & -5.946 & -3.392 & -7.223 & -7.223 & -7.520 \\
$A_{\mathbf{q}}^{y y}$ & -2.805 & -5.946 & -8.501 & -4.669 & -4.669 & -4.373 \\
$A_{\mathbf{q}}^{z z}$ & 9.798 & 11.893 & 11.893 & 11.893 & 11.893 & 11.893 \\
$A_{\mathbf{q}}^{x y}$ & 0 & 0 & 0 & -2.212 & 2.212 & 0 \\
\hline
\end{tabular}

Due to the sixfold rotation symmetry of the lattice, we can derive a general relation between dipole components in different directions:

$$
4 A_{\mathbf{q}(\varphi)}^{y y}=3 A_{\mathbf{q}\left(\varphi+60^{\circ}\right)}^{x x}+A_{\mathbf{q}\left(\varphi+60^{\circ}\right)}^{y y}-2 \sqrt{3} A_{\mathbf{q}\left(\varphi+60^{\circ}\right)}^{x y} .
$$

$\mathbf{q}(\varphi)$ denotes a wave vector along direction $\varphi$ in the Brillouin zone. From this formula we obtain relations for different points in the Brillouin zone: 


$$
\begin{aligned}
4 A_{\mathbf{q}_{3}}^{y y} & =3 A_{\mathbf{q}_{2}}^{x x}+A_{\mathbf{q}_{2}}^{y y}-2 \sqrt{3} A_{\mathbf{q}_{2}}^{x y} \\
4 A_{\mathbf{q}_{1}}^{x x} & =A_{\mathbf{q}_{2}}^{x x}+3 A_{\mathbf{q}_{2}}^{y y}-2 \sqrt{3} A_{\mathbf{q}_{2}}^{x y} \\
4 A_{\mathbf{q}_{1}}^{x x} & =A_{\mathbf{q}_{3}}^{x x}+3 A_{\mathbf{q}_{3}}^{y y}+2 \sqrt{3} A_{\mathbf{q}_{3}}^{x y} \\
0 & =3\left(A_{\mathbf{q}_{2}}^{x x}-A_{\mathbf{q}_{2}}^{y y}\right)+2 \sqrt{3} A_{\mathbf{q}_{2}}^{x y} .
\end{aligned}
$$

${ }^{1}$ Steiner M., Solid St. Commun 11, 73 (1972).

${ }^{2}$ Steiner M., Dachs H., Solid State Comun. 14, 841 (1974).

${ }^{3}$ M.Steiner, J. Villain and C.G. Windsor, Adv. Phys. 25, 87 (1976).

${ }^{4}$ Steiner M., Dorner B., Solid State Comun. 12, 537 (1973).

${ }^{5}$ H. Shiba, Solid State Commun. 41, 511 (1982).

${ }^{6}$ H. Shiba, N. Suzuki, J. Phys. Soc. Jpn. 51, 3488 (1982).

${ }^{7}$ H. Yamazaki, E. Soares, H. Panepucci and Y. Morishige, J. Phys. Soc. Jpn. 48, 1453 (1980).

${ }^{8}$ C. Pich and F. Schwabl, Phys. Rev. B 55, 3351 (1997). Fig. 4 of this figure is calculated for a dipole tensor $A_{0}^{x x}=$ -2.805 , which corresponds to a plate-like surface.

${ }^{9}$ M.Steiner M.J. Mikeska, Adv. Phys 40, 191 (1991).

${ }^{10}$ Babel D., Z. anorg. allg. Chem. 369, 117 (1969).

${ }^{11}$ C. Pich, Doctoral Thesis, Technische Universität München (1994).

12 M. Baehr, M. Winkelmann, P. Vorderwisch, M. Steiner, C. Pich, F. Schwabl, Phys. Rev. B 54, 12932 (1996).

${ }^{13}$ L. Bonsal and A.A. Maradudin, Phys. Rev. B 15, 1959 (1977).

14 J.M. Ziman, Principles of the theory of Solids, (Cambridge Press, 1969), p. $317 \mathrm{ff}$

${ }^{15}$ C. Pich and F. Schwabl, Phys. Rev. B 47, 7957 (1993).

${ }^{16}$ H. Yamazaki, E. Soares, H. Panepucci and Y. Morishige, J. Phys. Soc. Jpn. 47, 1464 (1979).

${ }^{17}$ M.H. Cohen, F. Keffer, Phys. Rev. 99, 1135 (1955).

${ }^{18}$ F. Keffer, in Encyclopedea of Physics, Vol. XVIII/2, edited by S. Flügge (Springer-Verlag, Heidelberg, 1966), p. 37ff 